\title{
Viscossuplementação como tratamento das alterações internas da articulação temporomandibular. Relato de casos*
}

\author{
Viscossuplementation to treat internal temporomandibular joint disorders. Case reports. \\ Daniel Bonotto ${ }^{1}$, Lílian Gonçalves Custódio ${ }^{2}$, Paulo Afonso Cunali³ \\ * Recebido do Ambulatório de Disfunção Temporomandibular e Dor Orofacial da Universidade Federal do \\ Paraná. Curitiba, PR.
}

\section{RESUMO}

JUSTIFICATIVA E OBJETIVOS: As formas de tratamento consideradas não invasivas para as alterações internas das articulações temporomandibulares (ATM) descritas na literatura são muitas, incluindo aconselhamento, farmacoterapia, fisioterapia e dispositivos interoclusais. No entanto, alguns pacientes tornam-se refratários aos tratamentos conservadores, sendo indicados procedimentos como artrocentese, artroscopia e cirurgias das ATM. A viscossuplementação é uma abordagem pouco invasiva, de baixo custo e com bons resultados em curto e médio prazo. O objetivo deste estudo foi discutir a técnica de viscossuplementação como tratamento das alterações internas da ATM, com relato de 2 casos clínicos com acompanhamento de 12 meses. RELATOS DOS CASOS: Duas pacientes com ar-

1. Mestre em Ciências da Saúde pela Pontifícia Universidade Católica do Paraná; Especialista em Disfunção Temporomandibular e Dor Orofacial pela Universidade Federal do Paraná; Professor do Curso de Odontologia da Universidade Positivo. Curitiba, PR, Brasil.

2. Especialista em Disfunção Temporomandibular e Dor Orofacial pela Universidade Federal do Paraná; Professora da Disciplina de Oclusão e Disfunção Temporomandibular da Universidade Federal do Paraná. Curitiba, PR, Brasil.

3. Doutor em Ciências pela Escola Paulista de Medicina da Universidade Federal de São Paulo; Mestre em Odontologia pela Universidade Camilo Castelo Branco; Coordenador do Curso de Especialização em Disfunção Temporomandibular e Dor Orofacial da Universidade Federal do Paraná. Curitiba, PR, Brasil.

Endereço para correspondência

Dr. Daniel Bonotto

Rua Padre Agostinho, 805 - Mercês

80430-050 Curitiba, PR.

Fones: (41) 9912-5608 - (41) 3322-1234

E-mail: danbonotto@ig.com.br tralgia das ATM e refratárias à tratamento conservador foram submetidas a uma infiltração semanal de hialuronato de sódio por três semanas. Observou-se melhora da dor e da amplitude de abertura mandibular nas duas primeiras semanas, resultado que se manteve constante ao longo de um ano de acompanhamento.

CONCLUSÃO: A viscossuplementação das ATM mostrou ser eficiente no controle da dor articular, melhorando também a função mandibular nos casos clínicos apresentados. Ensaios clínicos controlados com amostras significativas devem ser realizados para compreensão de sua real eficácia no tratamento das DTM.

Descritores: Ácido hialurônico, Articulação temporomandibular, Tratamento.

\section{SUMMARY}

BACKGROUND AND OBJECTIVES: There are several noninvasive treatments described in the literature for internal temporomandibular joint (TMJ) disorders, including counseling, drug therapy, physical therapy and interocclusal devices. However, some patients become refractory to conservative treatments, and procedures such as arthrocentesis, arthroscopy and ATM surgeries are indicated. Viscossuplementation is a less invasive, inexpensive and effective approach in the short and medium run. This study aimed at discussing viscossuplementation to treat internal TMJ disorders with 2 clinical case reports end 12-month follow-up.

CASE REPORTS: Two patients with TMJ arthralgia and refractory to conservative treatment were submitted to weekly infiltrations of sodium hialorunate for three weeks. Pain and mouth opening amplitude were improved in the first two weeks, and remained constant throughout the 12-month follow-up. 
CONCLUSION: TMJ viscossuplementation was an effective approach to control articular pain, also improving mandibular function in our cases. Controlled clinical trials with significant samples should be carried out to understand its actual effectiveness to treat TMD.

Keywords: Hialuronic acid, Temporomandibular joint, Treatment.

\section{INTRODUÇÃO}

Dentre as disfunções temporomandibulares (DTM) articulares, as incoordenações do complexo cabeça da mandíbula e disco articular, decorrem do colapso da função normal do disco sobre a cabeça da mandíbula pela incompetência dos ligamentos colaterais do disco e lâmina retrodiscal inferior. Nesse grupo das DTM articulares encontram-se os deslocamentos de disco com e sem redução. Essas disfunções muitas vezes estão associadas às alterações inflamatórias como sinovite, retrodiscite e capsulite ou alterações degenerativas como osteoartrose e osteoartrite ${ }^{1}$.

Em geral, o protocolo primário de controle das DTM prioriza as medidas mais simples, reversíveis e menos invasivas. Entretanto, como as disfunções intracapsulares muitas vezes são o resultado de doenças das superfícies articulares, ou seja, de alterações estruturais presentes, o tratamento conservador mostra-se algumas vezes ineficaz. Várias formas de tratamento para as disfunções internas da ATM são sustentadas pela literatura: repouso funcional, anti-inflamatórios não esteroides (AINES), dispositivos interoclusais, exercícios fisioterápicos de suporte, injeções intra-articulares de corticosteroide, artrocentese, artroscopia, cirurgia aberta da ATM, entre outras.

A viscossuplementação com injeção intra-articular de hialuronato de sódio (HS) - o sal de sódio do ácido hialurônico (HA) - foi primeiramente utilizado para tratamento de artrite traumática em cavalos de corrida $^{2}$, passando a ser usada em humanos para osteoartrite das grandes articulações como joelho, quadril e ombro. A partir de 1979, o HS começou a ser indicado nas alterações internas das $\mathrm{ATM}^{3}$. Desde então alguns estudos têm procurado avaliar a efetividade da técnica, bem como estabelecer um protocolo para sua utilização.

O objetivo deste estudo foi discutir a técnica de viscossuplementação como alternativa no tratamento das alterações internas da ATM através do relato de dois casos clínicos com acompanhamento de 12 meses.

\section{RELATO DOS CASOS}

Caso 1: Paciente do sexo feminino, 18 anos, com queixa de travamento mandibular e restrição de movimento durante abertura bucal com instalação há três meses, associada à leve sensibilidade ao final da abertura. Durante a anamnese, a paciente relatou história de estalido na ATM esquerda com início há um ano e interrupção após o travamento. No exame clínico observaram-se deflexão mandibular para esquerda, amplitude de abertura bucal de $25 \mathrm{~mm}$, limitação grave na lateralidade direita e leve sensibilidade à palpação na região pré-auricular esquerda.

A hipótese diagnóstica de deslocamento de disco sem redução na ATM esquerda foi confirmada por exame de ressonância nuclear magnética (RNM) (Figura 1). Foi realizada a terapia de viscossuplementação para ATM esquerda com a injeção intra-articular de $1 \mathrm{~mL}$ de HS. Após a infiltração foi realizada a manobra de manipulação mandibular na intenção de melhorar a relação do complexo cabeça-disco. No pós-operatório imediato, a abertura bucal aumentou para $32 \mathrm{~mm}$ com diminuição da gravidade da deflexão durante o movimento. Mais duas infiltrações foram realizadas com uma semana de intervalo entre cada sessão.

Após 60 dias do fim do tratamento, a abertura bucal mantida foi de $45 \mathrm{~mm}$ sem sintomatologia dolorosa para ATM esquerda durante a função mandibular. Com 12 meses de acompanhamento os resultados se mantiveram constantes. O exame de RNM realizado 6 meses após o fim do tratamento mostra o disco articular deslocado anteriormente sem redução, porém com melhor excursão da cabeça mandibular, se comparado com o exame anterior (Figura 2).

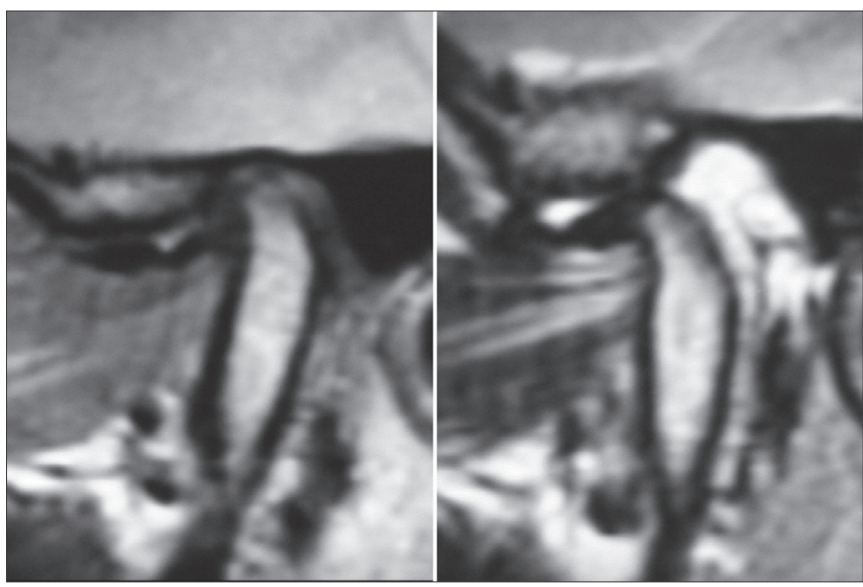

Figura 1 - Ressonância nuclear magnética em posição de boca fechada e aberta demonstrando deslocamento anterior do disco sem redução e restrição da excursão condilar. 


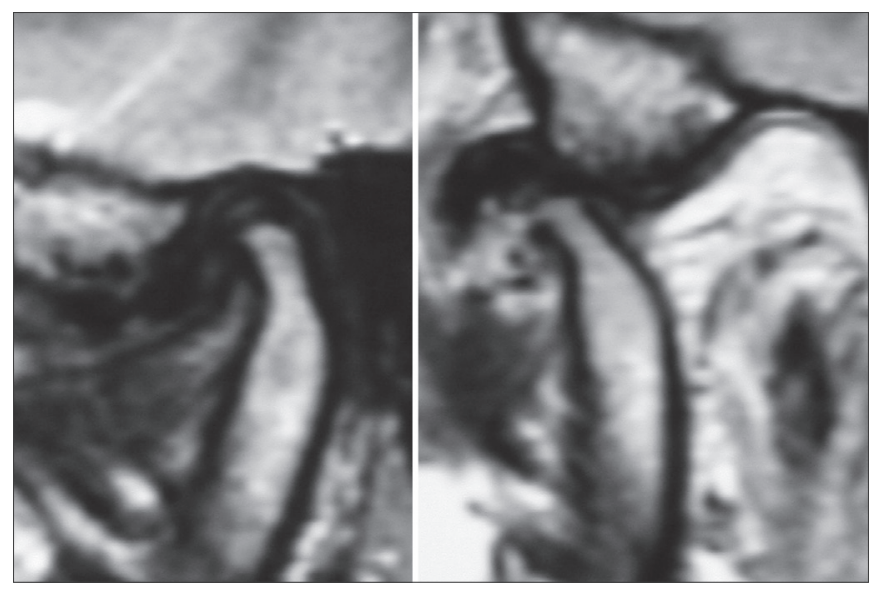

Figura 2 - Ressonância nuclear magnética, realizada 6 meses após término do tratamento, em posição de boca fechada e aberta demonstrando deslocamento anterior do disco sem redução e melhor amplitude de excursão condilar.

CASO 2: Paciente do sexo feminino, 23 anos, apresentava queixa de dor facial difusa bilateral de forte intensidade, desencadeada e exacerbada pela função mandibular, iniciada após acidente automobilístico, há 8 anos. Relatava ainda estalido na ATM esquerda e sensação de travamento durante abertura bucal. No exame clínico constatou-se sensibilidade à palpação na musculatura mastigatória e cervical e dor na ATM esquerda. Restrição da abertura bucal $(23 \mathrm{~mm})$ e dos movimentos excursivos da mandíbula foram observados, sendo essas limitações impostas pela dor.

A hipótese diagnóstica de artralgia por deslocamento de disco com redução para ATM esquerda foi confirmada por exame de RNM (Figura 3). A presença de dor muscular local secundária à artralgia foi considerada.
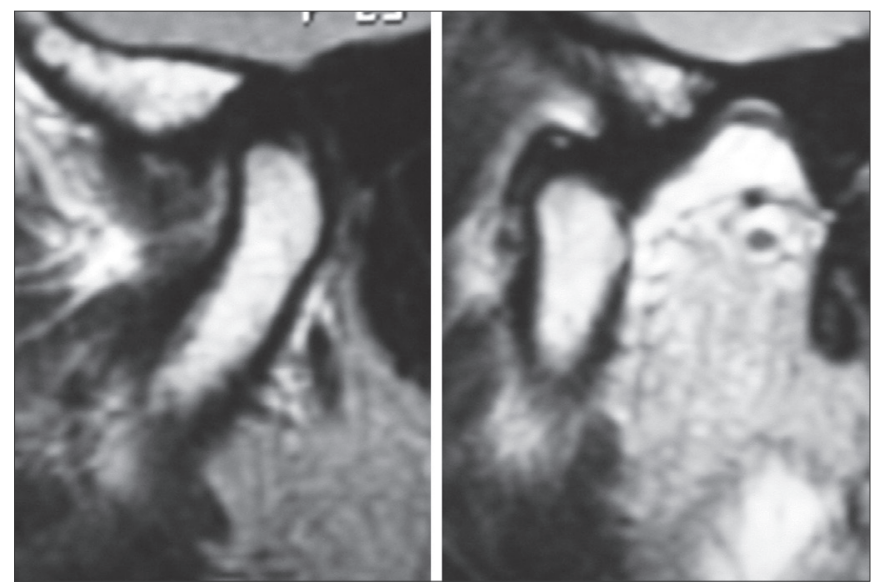

Figura 3 - Ressonância nuclear magnética em posição de boca fechada e aberta sugerindo deslocamento anterior de disco com redução.

Como fatores perpetuantes e contribuintes identificaram-se: hábitos parafuncionais de apertamento e mor- discamento de lábio, estresse emocional e limitação voluntária dos movimentos mandibulares, por "medo" do ruído articular.

Inicialmente, optou-se por medidas terapêuticas conservadoras: aconselhamento para mudança de hábitos orais, termoterapia, exercícios de coordenação e alongamento para musculatura mastigatória, medicação com AINES (etoricoxibe $120 \mathrm{mg}$ ) e dispositivo interoclusal. Após dois meses de acompanhamento semanal a paciente mostrou-se refratária ao tratamento, não apresentando melhora significativa para dor e função.

Foi realizada então a viscossuplementação da ATM esquerda e direita conforme a técnica descrita por Guarda-Nardini e col. ${ }^{4}$. Após a primeira infiltração já houve melhora quantitativa e qualitativa para dor e função. Sessenta dias após a terceira aplicação o quadro clínico manteve-se estável, com abertura bucal de $48 \mathrm{~mm}$, sem queixa de dor durante a função. Houve também redução da sensibilidade à palpação muscular e articular. Com 12 meses de acompanhamento os resultados se mantiveram constantes.

\section{Descrição da técnica (Figura 4)}

1. Marcação de um ponto $10 \mathrm{~mm}$ anterior ao trágus e $2 \mathrm{~mm}$ abaixo da linha trágus - borda inferior da órbita com lápis dérmico para indicar o local da infiltração para acesso ao compartimento superior da ATM. 2. Desinfecção com polivinil pirolidona iodo a $10 \%$ de toda região pré-auricular. 3. Anestesia local com infiltração intracapsular de $0,5 \mathrm{~mL}$ de lidocaína a $2 \%$ ou mepivacaína a $3 \%$ sem vasoconstritor. Introdução da agulha no ponto marcado atingindo o espaço supradiscal. 4. Infiltração de $1 \mathrm{~mL}$ de HS com seringa de $3 \mathrm{~mL}$ e agulha $0,7 \times 25 \mathrm{~mm}$ (22G). 5. Curativo removido em 24 horas.

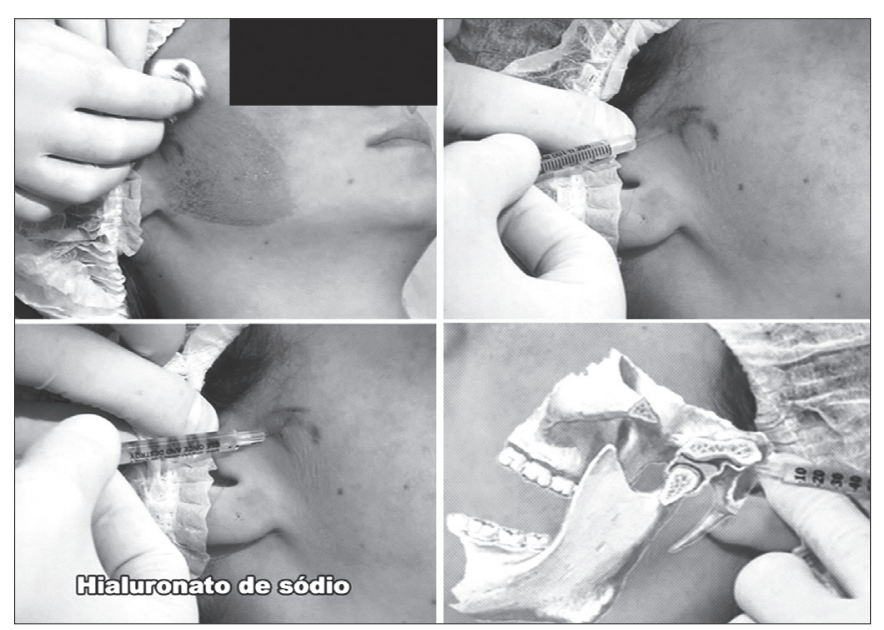

Figura 4 - Sequência clínica do procedimento de viscossuplementação. 


\section{DISCUSSÃO}

As articulações temporomandibulares são diartroses revestidas internamente por uma membrana que produz o líquido sinovial, que preenche o espaço articular superior e inferior. O líquido sinovial é responsável pela nutrição e lubrificação dos tecidos articulares, estando sua quantidade e qualidade diretamente relacionadas à saúde e à função articular ${ }^{5,6}$.

O HA é um mucopolissacarídeo ácido presente na substância fundamental dos tecidos animais. É formado por muitas unidades alternadas de ácido D-glucorônico e N-acetil-glicosamina, constituindo soluções gelatinosas altamente viscosas devido a sua elevada hidrofilia. O HA é o maior componente do líquido sinovial, tendo importante função na lubrificação dos tecidos articulares, devido ao seu alto peso molecular. Nas alterações inflamatórias e degenerativas das articulações a concentração e o peso molecular do HA estão diminuídos ${ }^{7}$.

A injeção do HS aumenta a concentração e o peso molecular de HA no líquido sinovial, o que estaria relacionado ao alívio da dor ${ }^{8}$. A liberação das zonas de aderências entre o disco articular e a fossa mandibular aumenta a mobilidade articular, permitindo melhor circulação do líquido sinovial. Prostaglandina E2 e leucotrieno B4 foram identificados no líquido sinovial de pacientes com dor articular, sugerindo que esses mediadores estão entre os fatores que geram dor articular ${ }^{9}$. Acredita-se que o efeito analgésico da viscossuplementação possa ocorrer pelo bloqueio de receptores e substâncias álgicas endógenas nos tecidos sinoviais.

Um mecanismo puramente mecânico pela interrupção do trauma causado pelo bloqueio mecânico do disco ou das zonas de aderência também foi sugerido, o que explica os efeitos da terapia a médio e longo prazo, pois enquanto o HA injetado permanece na articulação por apenas alguns dias, os resultados se mantém por meses ${ }^{10}$.

Comparando-se a viscossuplementação por HS com placebo, não se percebe diferenças estatísticas significativas em curto prazo na melhora de sinais clínicos das DTM. No entanto, há evidências de que o HS tenha efeito em longo prazo na melhora de sinais e sintomas em relação ao placebo $^{11}$.

O HS apresenta os mesmos efeitos benéficos que a infiltração com corticosteroide a curto e longo prazo na melhora dos sintomas e sinais clínicos das DTM ${ }^{12,13}$.

Os efeitos colaterais da técnica parecem ser de curta duração e autolimitantes ${ }^{5}$.
Não há na literatura uma indicação precisa para a viscossuplementação, porém parece consensual a utilização nos casos de alterações internas sintomáticas das ATM, principalmente nas que ocorre limitação da amplitude de movimentos.

Nos dois casos clínicos apresentados havia artralgia associada à restrição da mobilidade mandibular por alteração interna da ATM. A melhora objetiva e subjetiva das pacientes para dor e função foi condizente com os resultados apresentados por diversos autores.

\section{CONCLUSÃO}

A viscossuplementação com HS pode ser considerada uma medida terapêutica eficiente no restabelecimento funcional das ATM, a curto e médio prazo. Ensaios clínicos controlados com casuísticas significativas e com período de acompanhamento maior mostram-se necessários para avaliar a real eficácia da técnica de viscossuplementação e estabelecer um protocolo objetivo.

\section{REFERÊNCIAS}

1. Okeson JP, de Leeuw R. Differential diagnosis of temporomandibular disorders and other orofacial pain disorders. Dent Clin North Am 2011;55(1):105-20.

2. Butler J, Rydel NW, Balazs EA. Hyaluronic acid in synovial fluid. VI. Effect of intra-articular injection of hyaluronic acid on the clinical symptoms of arthritis in track horses. Acta Vet Scand 1970;11(12):139-55.

3. Kopp S, Wenneberg B. Effects of occlusal treatment and intraarticular injections on temporomandibular joint pain and dysfunction. Acta Odontol Scand 1981;39(2):87-96.

4. Guarda-Nardini L, Tito R, Staffieri A, et al. Treatment of patients with arthrosis of the temporomandibular joint by infiltration of sodium hyaluronate: a preliminary study. Eur Arch Otorhinolaryngol 2002;259(5):279-84.

5. Bertolami CN, Gay T, Clark GT, et al. Use of sodium hyaluronate in treating temporomandibular joint disorders: a randomized, double-blind, placebo controlled clinical trial. J Oral Maxillofac Surg 1993;51(3):232-42. 6. Sato S, Oguri S, Yamaguchi K, et. al. Pumping injection of sodium hyaluronate for patients with non-reducing disc displacement of the temporomandibular joint: two year follow-up. J Craniomaxillofac Surg 2001;29(2):89-93.

7. Listrat V, Ayral X, Patarnello F. Arthroscopic evaluation of potential structure modifying activity of hyaluronan (Hyalgan) in osteoarthritis of the knee. Osteoarthritis Cartilage 1997;5(3):153-60. 
8. Swann DA, Radin EL, Nazimiec M, et al. Role of hyaluronic acid in joint lubrication. Ann Rheum Dis 1974;33(4):318-26.

9. Quinn JN, Bazan NG. Identification of protaglandin E2 and leukotriene B4 in the sinovial fluid of painful, dysfunctional temporomandibular joints. J Oral Maxillofac Surg 1990;48(9):968-71.

10. Sato S, Ohta M, Ohki H, et al. Effect of lavage with injection of sodium hyaluronate for patients with nonreducing disk displacement of the temporomandibular joint. Oral Surg Oral Med Oral Pathol Oral Radiol Endod 1997;84(3):241-4.

11. Hepguler S, Akkoc YS, Pehlivan M, et al. The efficacy of intra-articular sodium hyaluronate in patients with reducing displaced disc of the temporomandibular joint. J Oral Rehabil 2002;29(1):80-6.

12. Kopp S, Wenneberg B, Haraldson T, et al. The short-term effect of intra-articular injections of sodium hyaluronate and corticosteroid on temporomandibular joint pain and dysfunction. Oral Maxillofac Surg 1985;43(6):429-35.

13. Kopp S, Akerman S, Nilner M. Short-term effects of intra-articular sodium hyaluronate, glucocorticoid, and saline injections on rheumatoid arthritis. J Craniomandib Disord 1991;5(4):231-8.

Apresentado em 05 de maio de 2011.

Aceito para publicação em 30 de agosto de 2011. 